\title{
AXPs \& SGRs: Magnetar or Quarctar?
}

\author{
Guojun Qiao ${ }^{1}$, Xionwei Liu ${ }^{1}$, Renxin $\mathrm{Xu}^{1}$, Yuanjie $\mathrm{Du}^{2}$, Jinlin $\mathrm{Han}^{3}$, \\ Hao Tong ${ }^{4}$ and Hongguang Wang ${ }^{5}$ \\ ${ }^{1}$ Department of Astronomy, Peking University, Beijing 100871, China, \\ email: gjn@pku.edu.cn, xiongwliu@163.com, r.x.xu@pku.edu.cn \\ ${ }^{2}$ Center for Space Science and Applied Research, CAS, Beijing, 100190, China \\ email: duyj@nssc.ac.cn \\ ${ }^{3}$ National Astronomical Observatories, CAS, Beijing 100012, China \\ email: hjl@nao.cas.cn \\ ${ }^{4}$ Xinjiang Astronomical Observatory, CAS, Urumqi, Xinjiang 830011, China \\ email: tonghao@xao.ac.cn \\ ${ }^{5}$ Center for Astrophysics, Guangzhou University, Guangzhou 510400, China \\ email: cosmic008@yahoo.com.cn
}

\begin{abstract}
The concept of a "magnetar" was proposed mainly because of two factors. First, the X-ray luminosity of Anomalous X-ray Pulsars (AXPs) and Soft Gamma-Ray Repeaters (SGRs) is larger than the rotational energy loss rate $\left(L x>\dot{E}_{\text {rot }}\right)$, and second, the magnetic field strength calculated from "normal method" is super strong. It is proposed that the radiation energy of magnetar comes from its magnetic fields. Here it is argued that the magnetic field strength calculated through the normal method is incorrect at the situation $L x>\dot{E}_{\text {rot }}$, because the wind braking is not taken into account. Besides, the "anti-magnetar" and some other X-ray and radio observations are difficult to understand with a magnetar model.

Instead of the magnetar, we propose a "quarctar", which is a crusted quark star in an accretion disk, to explain the observations. In this model, the persistent X-ray emission, burst luminosity, spectrum of AXPs and SGRs can be understood naturally. The radio-emitting AXPs, which are challenging the magnetar, can also be explained by the quarctar model.
\end{abstract}

\section{Introduction}

Anomalous X-ray Pulsars (AXPs) and Soft Gamma-Ray Repeaters (SGRs) have been generally recognized as neutron stars with super strong magnetic fields, namely magnetars. The primary properties of magnetars are:

1) The strength of magnetic field calculated from normal method is super-strong;

2) The X-ray luminosities are larger than the rotational energy loss rate, i.e. $L_{X}>\dot{E}_{\text {rot }}$;

3) The radiation energy comes from the energy of the magnetic field.

Here it is argued that: 1) The magnetic field can not be calculated from normal method when $L_{X}>\dot{E}_{\text {rot }}$;2) $L_{X}>\dot{E}_{\text {rot }}$ does not mean that the radiation energy is coming from the energy of the magnetic field; 3) Pulsed radio emission observed from some AXPs implies that it should be found another way to understand the observed phenomenons.

Following the arguments we present a model, quarctar, whose main properties are described below briefly.

\section{Do magnetars really exist?}

2.1. Is the magnetic field strength calculated from normal method correct?

When considering the following points, one finds that the method to calculate the magnetic field widely used in the literature is not suitable for AXP/SGR (Qiao et al. 2010). 
1) The energy loss is carried out not only by magnetic dipole radiation but also by wind;

2) Particle acceleration and radiation depend on the electric potential in the polar cap regions;

3) If the energy carry out by the high energy particles is larger than the dipole radiation, then the value of magnetic field calculated from the classical method, i.e., the rotational energy loss equals to the dipole magnetic radiation energy loss, $\dot{E}_{\text {rot }}=\dot{E}_{\text {dipole }}$, is incorrect. The wind braking should be taking into account (Tong et al. 2012). Probably, this is just the case of AXPs/SGRs.

\subsection{Anti-magnetar observations: challenge to magnetars}

There are observed anti-magnetar phenomena. For example, in PSR J1852+0040, $P=$ $105 \mathrm{~ms}, \dot{P}=(8.68 \pm 0.09) \times 10^{-18} \mathrm{~s} \mathrm{~s}^{-1}$, the surface magnetic field is $B_{s}=3.1 \times 10^{10} \mathrm{G}$, which is the weakest magnetic field ever measured for a young neutron star. Its X-ray luminosity is $L_{X}=5.3 \times 10^{33}(d / 7.1 \mathrm{kpc})^{2} \mathrm{erg} \mathrm{s}^{-1}$, while the rotational energy loss is $\dot{E}_{\mathrm{rot}}=3.0 \times 10^{32} \mathrm{erg} \mathrm{s}^{-1}$; thus $L_{X} / \dot{E}_{\mathrm{rot}} \simeq 17.7$ (Halpern \& Gotthelf 2010). This means that: $L_{x}>\dot{E}_{\text {rot }}$, which does not mean super-strong magnetic field at all! Beside that, some observations show that weak field "anti-magnetar" neutron star is still not ruled out for SN1987A (Manchester 2007; Gotthelf \& Halpern 2008).

\subsection{Radio observations: the difference between radio pulsars and magnetars does not originate from the difference of magnetic fields}

Previous observations mainly show that: (1) no radio emission from magnetars are observed; (2) the magnetic fields of magnetars are stronger than those of normal radio pulsars. Then it is generally believed that these differences are caused by the difference of the magnetic fields.

Recent observations show that all these two differences are confusing: some radio pulsars have stronger magnetic field than that of some AXPs (such as Hobbs et al. 2004; Kaspi \& McLaughlin 2005); radio emission from some AXPs are observed clearly after Xray flare (Halpern et al. 2005; Camilo et al. 2006; Lazaridis et al. 2008). These mean that the differences between radio pulsars and magnetars do not originate from the difference of magnetic fields strength.

\section{AXPs \& SGRs are quarctars?}

Instead of the magnetars, we suggested a quarctar model: a quark star with a crust in an accretion disk to account for the $\mathrm{X}$-ray and radio emission properties.

\subsection{Quark star with crust: X-ray emission}

It is assumed that crust of quark stars would be formed after a supernova explosion (e.g. Alcock et al. 1986). This kind of quark stars can not be observed as radio pulsars, but bare quark stars can be observed as radio pulsars (Xu et al. 2001). Therefore quark star with crust may be observed in X-ray bands. The pulse profiles in X-ray light curves should be wider and consistent with observations.

\subsection{Quark star with two polar holes: radio emission}

In the super flare, owning to the out-flow of high energy particles from the polar cap regions, two holes will form in the crust after some time. In this case, the polar cap regions become "bare", so that it can generate radio emission from the bared regions. Since the quark star lies in an accretion disk, later, the polar holes will be filled by the accreted matter, hence it becomes radio quite. This scenario is consistent with observations. 


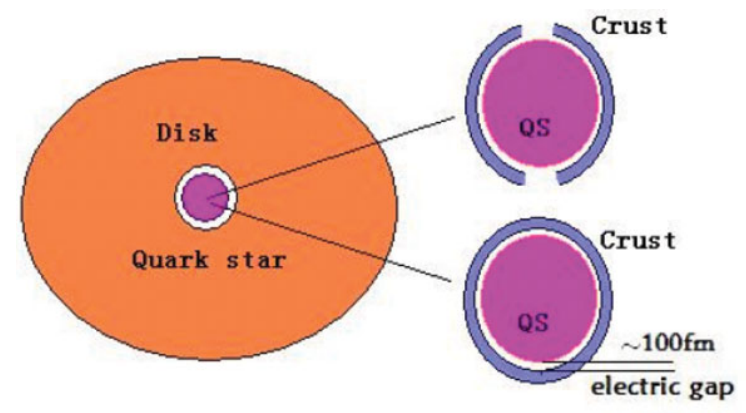

Figure 1. A quark star with a crust in an accretion disk, namely quarctar. Around the quark star there is a crust, in normal case, we can just observe radiation in X-ray bands; in the super flare, high energy particles flowing out from the pole cap regions, so there are two polar cap holes formed in the crust, in this case one can observe radio emission from polar cap regions.

\subsection{Energy source of the radiations}

Phase transition energy comes from the phase transition of normal matter to strange matter that takes place near the polar cap regions of the strange stars with a crust. For a magnetized star, the accreting material falls to the surface through the magnetic tube along the open field lines. The ions can be formed at the polar cap regions. The ions can be supported by the electromagnetic force. However, this balance can be destroyed easily, so that the normal matter can be transferred to the quark matter. This is a way to support the energy loss from the polar cap regions.

$\mathrm{Xu}$ et al. (2006) suggested that the energy of superflares of SGR can be supported by giant quakes in solid quark stars, which is a rich energy source for SGRs.

\section{Acknowledgements}

This work is supported by the National Basic Research Program of China (2009CB824800 and 2012CB821800), the NSFC (10935001, 10973002, 10833003, 11103021, 10821061, 10573002, 10778611, 10773016, 11073030) and the Key Grant Project of Chinese Ministry of Education (305001).

\section{References}

Alcock, C., Farhi, E., \& Olinto, A. 1986,ApJ, 310, 261

Camilo, F., Ransom, S. M., Halpern, J. P., et al. 2006, Nature, 442, 892

Gotthelf, E. V. \& Halpern, J. P. 2008, AIPC 983, 320

Halpern, J. P. \& Gotthelf, E. V. 2010, ApJ, 709, 436

Hobbs, G., Faulkner, A., Stairs, I. H. et al. 2004, MNRAS, 352, 1439

Kaspi, V. M. \& McLaughlin, M. A. 2005, ApJL, 618, L41

Manchester, R. N. 2007, AIPC 937, 134

Lazaridis, K., Jessner, A., Kramer, M., et al. 2008, MNRAS, 390, 839

Qiao, G. J., Xu, R. X., \& Du, Y. J. 2010, arXiv:1005.3911

Tong, H., Xu, R. X., Song, L. M., \& Qiao,G. J. 2012, arXiv:1205.1626

Xu, R. X., Zhang, B., \& Qiao, G. J. 2001, Astroparticle Physics, 15, 101

Xu, R. X.,Tao, D. J., \& Yang, Y. 2006, MNRAS 373, L85 